(Aus der inneren Abteilung des städtischen Krankenhauses Mainz.)

\title{
Über einige seltene Formen der Migräne.
}

Von

\section{Hans Curschmann.}

Kasuistische Mitteilungen über eine so häufige und vielgestaltige Erkrankung, wie die Migräne, haben nur dann einen wissenschaftlichen Zweck, wenn sie entweder für die Ätiologie und die Pathogenese des Leidens, überhaupt für allgemeine pathologische Fragen, etwas bedeuten oder ganz neue und ungewöhnliche Symptome und Ursachen schildern. Gerade die Pathogenese ist noch immer ein Streitobjekt. Wer die vorzügliche letzte Bearbeitung unseres Themas, die Migräne von E. Flata ${ }^{1}$ ), durchliest, wird sehen, dass noch immer - oder jetzt mehr als je - die grob lokalistischen Theorien gegen die vasomotorisch-sekretorischen streiten und dass in letzter Zeit aus den letzteren sich die Lehre entwickelt hat, die ich ebenfalls vor einigen Jahren schon vertrat, dass die Migräne, d. i. der hemikranische Kopfschmerz, nur die Teilerscheinung einer allgemeinen vasomotorischen, sekretorischen und trophisehen Neurose sein könne.

E. Flatau sieht die eigentliche Ursache der Migräne in Stoffwechselstörungen, die zu einem pathologischen „Neurometabolismus" führen, dem auch andere nervöse (vor allem vasomotorische und sekretorische) Störungen entstammen können.

Die eigentliche Ursache dieser Stoffwechselstörungen ist sicher keine einhejtliche. Wenn wir uns nach Analogien nmsehen, so werden wir unter manchen anderen die Epilepsie, die Hysterie oder noch besser eine eng umgrenzbare Krankheit wie die Tetanie anführen können, für die ganz das Gleiche gilt. Auch hier kann der sehr simple Symptomenkomplex des Karpopedalkrampfs und der Nervenübererregbarkeitssymptome durch eine grosse Reihe heterogener Auslösungsursachen hervorgerufen werden, unter denen nur die toxisch-infektiöse Noxe der Handwerkertetanie, die Bleiver-

1) Berlin, Jul. Springer. 1912. 
giftung, die Gravidität, die Menstruation, die Magenerweiterung, die operative Entfernung der Epithelkörper und viele andere zu nennen sind. Aber nur in der Mannigfaltigkeit der Ursachen ähnelt die Tetanie der Migräne, während der relativen symptomatologischen Einförmigkeit und Konstanz der ersteren eine unglaubliche Vielfältigkeit der hemikranischen Erscheinungen gegenübersteht.

Diese Fülle der Symptome, auch derjenigen, die nichts mit dem Schädelinnern und den Hirnnerven zu tun haben, heben die Hemikranie eben weit über das hinaus, was sie nach Möbius sein sollte, über eine durch primäre Veränderungen in der Hirnrinde hervorgerufene "Neuralgie".

Die von Möbius, Gowers u. a. verfochtene Anschaúung, dass die Schädigung des Parenchyms das Primäre, die der Zirkulation das Sekundäre sei, ist heute wahrscheinlich fast überall verlassen.

Die von Dubois und v. Möllendorf inaugurierte vasomotorische Theorie, durch Oppenheim, Pässler, Steckel, Buch, Flatau, Hans Curschmann u. v. a. weiter befestigt und ausgebant, hat ganz entschieden das Feld behauptet.

Mit Recht bedient sich E. Flatau bei der Beweisführung für die vasomotorische Pathogenese der Migräne auch meiner Beobachtungen über Hemikranie und Angina pectoris vasomotoria. ${ }^{\text {i }}$ )

Ich habe gezeigt, dass diese generalisierte vasomotorische Neurose Nothnagels in zahlreichen Fällen mit typischer Hemikranie im Anfall verbunden ist (ebenso wie die Angina pectoris vasomot. ja auch mit halbseitiger Amblyopie, mit objektiv nachweisbarem Spasmus der Art. retinae, mit halbseitiger Vertaubung und Schwindel, mit Urina spastica, mit krisenartigen spastischen Zuständen der vom N. splanchnicus versorgten Bauchorgane und Arterien [vgl. die Blutdrucksteigerung im Anfall!] und anderen vasomotorisch, spez. konstriktoriseh bedingten Erscheinungen verknüpft sein kann).

In einem dieser Fälle gelang es sogar, wie im Experiment, durch Eintauchen der Hände in kaltes Wasser zuerst den Angiospasmus der Hände, dann Angina pectoris-Grefühle und schliesslich einen mit Flimmerskotom und Erbrechen einhergehenden halbseitigen Kopfschmerz hervorzurufen; die Anamnese hatte ergeben, dass Pat. auf eiskaltes Wasser stets in dieser Weise reagiere.

Hier war die Annahme, dass die Ursache der Hemikranie ebenfalls, wie die Gefässkrämpfe der Finger und die vasomotorische Angina pectoris, eine vasokonstriktorische Erscheinung sei, so naheliegend, dass man sie fast als bewiesen ansehen kann. Auch sprechen das

1) Deut. Zeitschr. f. Nervenheilkde. Bd. 38, 1910, S. 211. 
gleichzeitige Anftreten von Migräne und intermittierendem Hinken (Goldbladt, Sterling) in demselben Sinne.

Ohne nun auf die übrigen, meist wenig glücklichen Theorien der Migränepathogenese (z. B. von Bonnier, Lévi, Spitzer, Deyl und Plavec, Schüller u. v. a) eingehen zu können, möchte ich in den folgenden Mitteilungen neben einigen symptomatologischen Seltenheiten hauptsächlich Beispiele für die eben wiedergegebene These bringen, dass die Migräne, d. i. der halbseitige Kopfschmerz mit seinen obligaten cerebralen Ausfalls- und Reizsymptomen, bisweilen und wohl nicht selten nur die Teilerscheinung einer allgemeinen vasomotorischsekretorischen Neurose ist, und dass in manchen Fällen die peripheren Symptome der letzteren Genese recht stark, den Cerebralsymptomen völlig koordiniert auftreten können.

Diese peripheren Symptome können mit der Zeit immer intensiver werden und schliesslich sogar den hemikranischen Kopfschmerz völlig substituieren, wie ich das in folgendem, bereits einmal mitgeteilten Falle zeige.

Eine 48jähr. Dame, schwer hereditär belastet, litt bis zur Menopause stark an typischer Migräne, besonders während der Menses, dazwischen an anfallsweise auftretenden Schleim-Membrankoliken des Dickdarms. Seit der Menopause werden diese Anfalle substituiert durch allmonatliche leichte Depressionen mit anginösem Schmerz in der Magengrube und dem Herzen mit heftiger Angst und totalem "Absterben" der Beine und geringeren Angiospasmen der. Finger, an denen dann nach jedem Anfall einige Fingernägel partiell absterben oder einreissen. Ein weiterer erst kürzlich beobachteter Fall zeigt ebenfalls diese Substitution der „Kopfmigräne" durch die "Bauchmigräne".

Fall 1. L. M., 19 Jahre, Dienstmagd. Mutter litt sehr viel an Nervenkopfweh mit regelmässigem Erbrechen, ein Bruder leidet ebenfalls an Migräne.

Pat. begann schon mit 7-8 Jahren an Migräne zu leiden: Das Leiden begann mit Anfällen von meist streng linksseitigem Kopfweh (ohne Flimmern), mit Erbrechen und Leibschmerzen, die auf beiden Seiten des Unterleibes - nicht in der Magengegend - sassen und mit Durchfällen endigten.

Das Leiden repetierte wechselnd in der Woche 2-3 mal, manchmal viel seltener, keine menstruelle Beeinflussung.

Seit ca. $1 \mathrm{Jahr}$ begann nun ein auffallender Wechsel der Beschwerden: Die der Pat. wohlbekannten "Anfälle" verliefen nicht mehr mit Kopfschmerzen, sondern lokalisierten sich stets und ausschliesslich in beide Seiten des Leibes, ziemlich tief im Unterleib. Die Schmerzen sollen „momentan", sehr rasch, ohne Vorboten eintreten, bisweilen mit einem diarrhoischen Stuhl verlaufen, meist aber mit mehrmaligem Erbrechen von galligem Schleim. - Der einzelne Anfall soll bisweilen viele Stunden lang dauern, bisweilen nur $1 / 4$ Stunde (die früheren typischen Hemikranieanfälle dauerten ca. 1 Tag). 
Während des Anfalls von Leibschmerz habe sie auch vermehrten Ansfluss (aus der Vagina) von gelblich-grünem Sekret, der in anfallsfreien Zeiten bei ihr vollständig fehlt. Keịn Anschwellen der Brüste wäbrend des Anfalls. Kein vermehrter Urindrang dabèi.

Bisweilen ist der „Anfall" mit Herzklopfen begleitet. *Häufig bemerkt sie auch im Anfall ein "Absterben" der Hände, die kalt, taub und bewegungsunfähig werden.

Auch ausserhalb des Anfalls leide sie öfters - - besonders nachts an Einschlafen der Hände und Füsse.

Während des Anfalls überläuft es sie ganz kalt; sie müsse dann stark kalt schwitzen.

Sofort nach Beendigung des Anfalls seien Appetit und Stuhl wieder ganz normal.

Der objektive Befund ergab - in anfallsfreier Zeit - nichts Besonderes. Leichte Anämie. Keine objektiven Veränderungen am Herzen. Puls regelmässig, weich. Blutdruck im Anfall $130 / 80 \mathrm{~mm} \mathrm{Hg} \mathrm{Riva-Rocci,}$ in anfallsfreier Zeit $115-110 / 70 \mathrm{~mm} \mathrm{Hg}$.

Lungen und Bauchorgane ohne Befund.

Auch das Nervensystem bis auf mässige Reflexsteigerung und Dermographismus $0 . \mathrm{B}$.

Urin 0. B.

Auf Bettruhe und Chinin in 3 Wochen nur ein ,Anfall", der mit kurzem heftigen Leibschmerz and Durchfall, ohne Erbrechen and ohne alles Kopfweh verlief. Die Untersuchung bestätigte, dass Pat. direkt nach dem Anfall einen starken gelben Fluor zeigte, der vorher nicht vorhanden war. Mikroskopisch: Keine Gonokokken, keine Vermehrung der eosinophilen Zellen.

In diesem jugendlichen Alter ist eine derartige Umformung einer Migräne jedenfalls sebr selten und darum bemerkenswert, zumal sie durch keinerlei Veränderung der Genitalfunktion (vgl. die Rolle der Climax im vorigen Fall) veranlasst wurde.

Hervorzuheben sind auch in diesem Falle die zahlreichen extracerebralen Beschwerden ausser dem Erbrechen, die Diarrhöen, der Fluor, die vasokonstriktorischen Anfälle an Händen und Füssen und die koinzidierenden Herzbeschwerden.

Beilänfig möehte ich übrigens bemerken, dass derartige Substitutionen nicht nur beim einzelnen Individuum, sondern auch bei der „Familienmigräne" vorkommen. Ich habe öfters gesehen, dass Kinder meist kurz vor der Pubertät an heftigen Bauch- oder Magenkoliken anscheinend ganz unsicherer Genese, meist mit starkem Erbrechen oder Diarrhöen verbunden, litten, die in oft unregelmässigen Zeitabschnitten wiederkehrten. Die Mütter litten ausnahmslos an schwerer Hemikranje typischer Art (Kopfweh, Erbrechen usw.), ebenso zahlreiche weibliche Blutsverwandte.

Die ganze Art des Auftretens der „Koliken“. der Kinder, das menstruelle Rezidivieren bei Mädchen, das ganz dem Typ der men- 
struellen Migräne der Mutter entsprach, liessen die "Bauchkoliken“ als sichere larvierte oder substituierte "Bauchmigräne" ansprechen, die in der zweiten Generation ihren Charalkter in dieser eigentümlichen Weise geändert hatte.

Therapentisch und prognostisch ist die Kenntnis solcher Fälle wichtig!

Sie geraten heutzutage gar zu leicht in die Gefahr, appendektomiert zu werden.

Auf die Eigenart der Kindermigräne, insbesondere die ersten Anfälle des betreffenden Pat., möchte ich am Ende dieser Arbeit nochmals eingehen. Das Kapitel bedarf dringend einer kasuistischen Bereicherung, die womöglich auch dem.Praktiker zugänglich sein muss. Denn unglaublich oft wird die infantile Migräne verkannt - und misshandelt.

Doch ich möchte nun zu unserem eigentlichen Thema, den vasomotorischen und sekretorischen paroxysmalen und intervallären Symptomen der Migräne zurückkehren und einige Fälle mitteilen, die bisher noch nicht beschriebene Erscheinungen dieser Art zeigten.

Ich beginne mit einem Fall von Migränegalaktorrhoe extra graviditatem bei einer sonst fast reinen Schwangerschaftsmigräne.

Fall 2. 29jähr. Dame, Arztgattin, Mutter von 3 Kindern.

Vater litt in der Jugend an ziemlich seltener typischer Migräne, Mutter litt an Diabetes und Hysteria gravis. Pat. ist stets gesund gewesen, ist gut begabt, insbesondere optisch, auch musikalisch.

Seit ihrer Kindheit leidet die sonst sehr vernünftige Frau an der Neigung zu nächtlichen Depressionen und Ängstlichkeit.

Sie litt früher niemals an Migräne. Während der ersten Gravidität in den ersten Monaten Erbrechen, keine Cephalea; während einer zweiten Gravidität, die am Schlnss des vierten Monats durch Trauma mit Abort endete, ebenfalls keine Migräne. Bei der dritten Gravidität erfolgten im zweiten bis dritten Monat unter gleichzeitigem häufigen Schwangerschaftserbrechen 3 Anfälle von typischer Hemikranie, und zwar während einer Zeit, die mit Erregungen und diätetischen Fehlern einherging. Die Anfälle dauerten ca. einen halben Tag und wurden durch Bettruhe and Aspirin beendet. Im Beginn des Anfalls - ca. 2 Stunden lang - sah Pat. alle Gegenstände, z. B. auch die Personen, auffallend verkleinert, wie in weiter Ferne, „als ob sie sie umgekehrt durch das Opernglas sähe"; zugleich bemerkte sie, dass die rechte Hälfte der gesehenen Objekte verdunkelt und undeutlich war.

Nach Beendigung dieser Gravidität (gesundes Kind ${ }^{1}$ )) keine Migräne mehr, auch nicht während einer vierten, sehr leicht zu ertragenden Gravidität.

1) Das Kind, ein gesundes, kräftiges Mädchen ohne sonstige Spuren der Nervosität, intelligent, aber nicht frühreif, leidet bemerkenswerter Weise seit 
Etwa vor einem Jahr hatte Pat. sehr abgenommen, fühlte sich elend, schlief schlecht und hatte allerlei häusliche Erregungen. Die Periode war normal, hatte nicht ausgesetzt. Damals trat plötzlich ein ziemlich schwerer Anfall von linksseitiger Migräne auf. Auch in diesem Anfall kehrte das oben beschriebene eigentümliche Fernsehen wieder, verbanden mit dem rechtsseitigen hemianopischen Skotom.

$\mathrm{Zu}$ gleicher Zeit kam es zu einer Anschwellung der Mammae, zu dem der Pat. wohlbekannten Gefühl des Spannens und Ziehens in der Brust "genau wie vor dem Stillen" und zur spontanen ziemlich reichlichen Sekretion aus der Brustdrüse; das Sekret entsprach seiner Beschaffenheit genau dem Colostrum während der Graviditäten. Die Sekretion der Brust danerte genau so lange, wie der schwere Migräneanfall, der mit Übelkeit und anch Erbrechen einherging, ca. 11/2 Tage lang.

Natürlich vermutete Pat., gravide zu sein. Sie war es aber nicht, wie das regelmässige weitere Menstruieren und die weitere Entwicklung der Dinge lehrte: sie wurde nicht schwanger. Auch ein etwaiger „kleiner" Abort in einem frühen Monat ist durch genaue ärztliche Beobachtung auszuschliessen.

Seit dieser Zeit hat Pat. noch einige kurze Anfälle von Hemikranie, die sie aber durch Aspirin koupieren konnte; zur Mikropsie, Hemianopsie und auch zur Sekretion von Colostrum ist es nicht mehr gekommen.

Im Oktober 1914 warde Pat. nun wirklich gravida. Das allererste Zeichen, das auch den Verdacht der Gravidität erregte, war wieder ein schwerer Anfall von Migräne (wieder mit Fernsehen und Hemianopsie verbunden), diesmal obne Anschwellang und Sekretion der Mammae. Es war seit dem obigen Migräneanfall mit Sekretion der Brüste, aber ohne Gravidität ca. $1 \frac{1}{2}$ Jahre ohne jeden Migräneanfall verstrichen.

Die Hemikranie kehrte bei der nun sich normal entwickelnden Gravidität nur selten wieder. Ein einziger typischer und heftiger Anfall mit allen Augensymptomen erfolgte noch einmal ganz kurz, ca. 2 Tage vor der (normalen) Entbindung. Dann folgte ungestörte, gänzlich migränelose Laktation.

Die Untersuchung ergab ausser einer gewissen Anämie und Asthenie keine organischen Veränderungen. Das Nervensystem, insbesondere der Augenbefund, waren im freien Intervall völlig intakt; im Urin kein Eiweiss und Zucker.

Epikrise: Eine familiär speziell belastete Frau, die aber noch niemals Migrä̀neanfälle gehabt hatte, wird während ihrer dritten Gravidität zum ersten Mal von typischen, ziemlich schweren Anfällen befallen, deren Augensymptome in Mikropsie and hemianopischer Gesichtsfeldeinschränkung bestanden. 3 Jahre später erleidet sie wieder dieselben Anfälle mit derselben eigentümlichen Augenaura, diesmal, ohne gravide zu sein. Dabei tritt eine Anschwellung der Mammae und starke Colostrumsekretion auf, die sofort nach

dem zweiten Jahr an sehr seltenen Anfällen von plötzlichem Erblassen, bisweilen leichter Ohnmacht mit Erbrechen; auch bei Eisenbahn- und Wagenfahrten wird es leicht bleich und klagt über Übelkeit. Kopfweh klagt das Kind - noch - nicht. 
Beendigung "des Anfalls verschwindet. Eine weitere vierte Gravidität verlâufft wieder im ersten Beginn und in den letzten Tagen mit der geschilderten Migräne.

Der Fall ist, wie ich bei Durchsicht der Literatur sehe, eln absolutes Unikum bezüglich der im Migräneanfall auftretenden Sekretion der Brustdrüsen. Ich finde unter den zahlreichen paroxysmalen Störungen der Sekretion, wie sie bei der Migräne beobachtet werden, dies Symptom noch keinmal vermerkt.

Analoge Vorgänge sind auch bei der "Schwester der Migräne“, der Epilepsie, m. W. nicht geschildert worden, wohl aber bei der Tabes dorsalis. Siding und de la Camp haben als grosse Seltenheit Fälle von krisenartiger Galaktorrhoe und schmerzhafter Schwellung der Mammae bei tabischen Frauen beschrieben. Diese Mammakrisen sind jedenfalls weit seltener als die Clitoriskrisen; ich habe nie Derartiges gesehen.

Eine besondere Bedentung gewinnt die Migränegalaktorrboe in unserem Falle dadurch, dass diese Migräne ursprünglich ein reines Produkt der Gravidität war, jedenfalls in voller Ausbildung, insbesondere der eigentümlichen Augensymptome, nur während Schwangerschaften auftrat. Es muss als eine ganz eigentümliche Assoziation bezeichnet werden, dass die Hemikranie das einzige Mal, wo sie extra graviditatem auftrat, zu einem isolierten Schwangerschaftssymptom, der Schwellung der Brüste und starker Milchabsonderung, führte. Es liegen in diesem Falle also ganz enge Beziehungen zwischen der Funktion des Genitalapparats bzw. des ihm vorstehenden vegetativen Nervensystems und der Hemikranie vor, dergestalt, dass die zufällige Entstehung eines Migräneanfalls ausserhalb der Schwangersehaft in reziproker Weise wieder eine partielle Veränderung des Genitalsystems, nämlich die Milchsekretion der Brüste, hervorrief, die in dieser Ausgiebigkeit nur an eine bestehende Gravidität gebunden zu sein pflegt. Man kann hier direkt von einer gegenseitig wirkenden Reflextätigkeit sprechen.

Übrigens ist auch die reine Graviditätsmigräne an sich schon ein äusserst seltenes Ereignis. Wie bei der Epilepsie, so sieht man bekanntlich auch bei der Migräne weit häufiger eine Verminderung, ja vollständiges Aufhören der Anfälle während der Schwangerschaft und des Stillens; ich habe das besonders ausgesprochen bei Fällen von reiner Menstruationsmigräne, die ja recht häufig ist, gesehen.

Flatau und aụch Möbius erwähnen die Gravidität als Ursache der Migräne überhaupt nicht; ich habe auch sonst in der Literatur nichts über diesen Punkt gefunden. Es ist also ein bemerkenswertes 'Zusammentreffen, dass auch hierin Migräne und Epilepsie sich ähneln. 
Bekanntlich sind Fälle von Epilepsie, die nur während der Schwangerschaften rezidivieren, ebenfalls äusserst selten. Ich habe erst einen derartigen Fall gesehen und beschrieben.

Einen weiteren, bezüglich seiner trophisch-vasomotorischen Symptome der Peripherie bemerkenswerten Fall von Spätmigräne beobachtete ich in Gestalt eines 42jähriges Arztes.

Fall 3. Dr. $\mathrm{X}$, prakt. Arzt. Familienanamnese ohne spezielle Belastung, keine Migräne in der Familie, aber Mutter an Hirnsklerose gestorben, Vater in höherem Alter senil dement. X. war stets gesund, sehr robuste, arbeitsfreudige Natur. Seit Jahren stark überarbeiteter Kassenarzt. Kein Potus, kein Nikotinabusus, nie Lues. Heirat mit nervöser Frau. Seit einigen Jahren nach Erregung und viel Arbeit Extrasystolie des Herzens.

Seit ca. 5 Jahren typische Migräneanfälle leichteren Grades, alle paar Monate auftretend mit rechtsseitigem neuralgischen Kopfweh, Übelkeit, bisweilen Erbrechen. Dauer ca. 12 Stunden.

Vor 2 Jahren änderten und verschlimmerten sich nun diese Anfälle; Pat. schildert sie so: Er erwacht morgens schon mit dem Vorgefühl des Anfalls, dumpfem, wüstem Kopf. Im Laufe des Vormittags während der Praxis Verschlimmerung, schwerer rechtsseitiger Kopfschmerz, Übelkeit, regelmässiges Erbrechen. Nach dem Erbrechen, meist nach einem kurzen Mittagsschlaf, bekommt er äusserst heftiges Jucken in der linken Hohlhand und am linken vierten Finger, dasselbe auch am linken Fuss, besonders an der vierten Zehe. Dann treten ganz rasch an diesen Stellen je eine oder mehrere linsen-bis pfennigrosse, ziemlich schlaffe Blasen auf, die mit serösem, bisweilen leicht hämorrhagischem Inhalt gefüllt sind. Die Blasen trocknen meist ein, nur selten platzen sie auf. Mit dem Aufschiessen der Blasen versehwindet meist auch das Jucken. Damit ist auch der Migräneanfall meist beendet. Während eines ganzen Jahres traten diese Migräneanfälle, jedesmal mit Jucken und Aufschiessen von Blasen an der Haut der genannten Stellen verbunden, álle paar Wochen - je nach Überanstrengung auch öfter - auf und verliefen jedesinal in ganz stereotyper Weise.

Ich habe selbst die Blaseneruption häufig gesehen: es waren schlaffe, mit hămorrhagischem, serösem Inhalt gefüllte Blasen von Linsen- bis Pfenniggrösse, von etwas gerötetem Hof umgeben. Je eine befand sich in der Mitte der linken Hohlhand, eine an der Streckseite der Mitte des linken Goldfingers, je zwei an beiden Seiten der vierten Zehe des linken Fusses. Nachdem diese Blasen so häufig rezidiviert waren, haben "sie jetzt eine oberflächliche, etwas pigmentierte Narbenbildung hinterlassen.

Im äbrigen war der Pat. völlig gesund und robust, der-Urin frei von Eiweiss und Zucker, das Nervensystem frei von organischen Veränderungen und ohne alle funktionell-nervösen Züge.

Diese Form der Migräne mit stereotyper Blaseneruption an ebenso stereotypen Stellen wurde nun plötzlich durch eine schwere oft inzidierte Nackenphlegmone, an der Pat. im Oktober bis November 1914 litt, beseitigt. Sie ist seitdem nicht wiedergekehrt (seit ca. 8 Monaten!). Auch die ,gewöhnliche" Migräne ist wesentlich seltener und milder :geworden, trotzdem 
$X$. durch die dazutretende militärische Beschäftigung angestrengter ist als je.

Der Fall ist bezïglich des regelmässigen Auftretens der Blaseneruption an ganz bestimmten distalen Teilen gleichseitiger Extremitäten in einer ganz bestimmten Phase des Migräneanfalls (nach dem Höhepunkt, nach dem Erbrechen) sehr bemerkenswert und eine ausgesprochene Seltenheit. Die Neigung zu Neurodermatosen (Oedema fugax, Erythemen, Herpes, Urtiearia verschiedener Form) ist bei Hemikranischen im Intervall augenscheinlich wesentlich grösser und häufiger als im Anfall. Es entspricht das ja ganz Flataus und auch meiner Auffassung, die in der Migräne (in vielen Fällen) nur das ein e Symptom einer vasomotorisch-sekretorischen Neurose - oft ganz verschiedenartiger Form - erblickt. Flatau erwähnt eine ganze Reihe von solchen intervallären trophischen und sekretorisehen Erscheinungen, dagegen nur auffallend wenige Fälle, die im Anfall derartige Symptome mit Regelmässigkeit zeigten. Gerade das Symptom der regelmässig im Anfall rezidivierenden Blasenbildung (mit allerlei Parästhesien verbunden) finde ich in der Literatur überhaupt noch nicht beschrieben, während Flatau über drei Fälle berichtet, die interparoxysmal an solchen Blaseneruptionen litten. Was von vasomotorisch-trophischen Veränderungen paroxysmaler Natur bekannt ist, beschränkt sich auf lokale Asphyxie, wie ich sie im Hemikranie-Angina pectoris vasomotoria-Anfall bereits erwähnt habe, Erythromelalgie(?) (Möbius) und Hyperhidrosis der Extremitäten. Flatau betont mit Recht, wie auffallend viel häufiger die paroxysmalen Störungen von seiten des sympathischen prävertebralen Gangliensystems, das den Eingeweideapparat versorgt, seien, als diejenigen von seiten des vertebralen Systems.

Es ist in unserem Fall in Berücksichtigung dieser Tatsache besonders bemerkenswert, dass bei ihm alle paroxysmalen Störungen im . Bereiche des ersteren, also des Herzens, der Bancheingeweide (abgesehen rom Erbrechen) und der Blase, völlig fehlten.

Die - einstweilige - Heilung dieser eigenartigen Hemikranie durch eine interkurrente sehwere Eitexinfektion verdient endlich auch einige Beachtung. Bei der Migräne macht man diese Erfahrung relativ selten; bei der Epilepsie ist sie ja recht bekannt und anch schon Ausgangspunkt therapeutischer Bestrebungen gewesen.

Fall 4. In ein ganz anderes Kapitel gehört nun der folgende Fall: Stereotyp halluzinatorische Augenmigräne.

Katharina St., 35jährige verheiratete Näherin.

Vater an Schwindsucht $\dagger$, Mutter leidet an "Nervenkopfweh", wahrscheinlich auch ein Bruder. 
Als Kind gesund, angeblich gut gelernt; schon als Kind habe sie öfters an leichten Kopfschmerzen über den Augen gelitten ohne Nausea, ohne Erbrechen. Sie lernte nähen, habe die Augen besonders durch nächtliches Nähen bei Licht überanstrengt. Mit 15 Jabren Menses, dabei heftige Leibschmerzen und Krämpfe in den Händen mit eingeschlagenen Fäusten. Damals noch keine menstruelle. Migräne.

Mit 17 Jahren Heirat. In den ersten Jahren bestanden noch die. leichten früheren Kopfschmerzen weiter. Während aller Graviditäten Erbrechen vom ersten Monat an, bei der vierten Gravidität auch „Blutbrechen".

Damals, also Mitte der zwanziger Jahre, Verschlimmerung der Kopfschmerzen während jeder Menstruation. Seit dieser Zeit beginnt das Kopfweh (ibber beiden Augen) mit Flimmern vor den Augen; dabei habe sie, wenn die Schmerzen besonders schlimm waren, alles doppelt gesehen, bisweilen auch eine Verdunkelung des einen Gesichtsfeldes bemerkt. Die verdunkelte Partie eines Gegenstandes zeigte eine trübe, rötliche Schattierung und unscharfe Begrenzang. Der Anfall gehe seitdem stets mit Erbrechen einher.

Seit 5 Monaten habe sich ihr Leiden sehr versehlimmert: Der Anfall beginne jetzt besonders leicht morgens früh, direkt aus dem Schlaf herans, mit Stechen über den Augen, Hämmern auf dem Scheitel. Bevor der Kopfschmerz seinen Höhepunkt erreicht, hat sie ganz regelmässig folgende sich stets wiederholende Angenerscheinungen: Sie sieht vor sich "verzerrte Gesichter", Fratzen meist mit irgendwelchen Verstümmelungen, namentlich zerfressener Nase oder verstümmelten Ohren. Bei diesen Fratzen ist die linke Seite röter, dunkler und verschwommener als die rechte, "wie in einer Wolke".

Auf Befragen gibt sie mit Bestimmtheit an, dass nicht nur diese halluzinierten Fratzen, sondern auch die Gesichter veritabler Menschen ihrer Umgebung diese "Schatten" auf der linken Hälfte zeigten. Auch sollen diese Gesichter ihrer Umgebung im ganzen undeutlicher und verschwommener aussehen und bisweilen auch Verunstaltungen oder Verstümmelungen (wenigstens in Andeutungen) zeigen, allerdings lange nicht so grotesk als die der rein halluzinierten Gesichter (Pat. unterscheidet im Anfall scharf und bewusst zwischen realen und hallazinierten Bildern).

Bisweilen begleitet diese Erscheinungen auch horizontales Doppelt-, selbst Dreifachsehen.

Das Fratzensehen tritt stets im Beginn des Anfalls auf, dauert ca. 10 Minuten und wiederholt sich dann öfters im Lauf der Kopfschmerzen. Der ganze Anfall dauert ein bis mehrere Tage. Die geschilderten Augensymptome nehmen allerdings im Laufe des Anfalls sehr ab. Sowie sie erbrechen kann, ginge der Anfall zurück, die Augenerscheinungen hörten dann ganz auf.

Während des Anfalls soll ihre rechte Gesichtshälfte "feuerrot" aussehen, besonders das $\mathrm{Ohr}$, die Schläfenadern. sollen dick anschwellen.

Der körperliche Befund bei der Kranken war so gut wie negativ. Es fehlten objektive Veränderungen am Herzen und den Gefässen. Die Arterien waren weich, der Blutdruck (in anfallfreier Zeit) normal, $110 \mathrm{~mm} \mathrm{Hg}$ (Riva-Rocei).

Urin ohne Alb., ohne Zucker. 
Schläfenarterien und Carotiden beiderseits gleich.

Nervenbefund gleichfalls frei von organischen Veränderungen. Pupillen gleich, mittelweit, von normaler Reaktion bei Licht and Konvergenz.

Der Augenbefund in anfallfireier Zeit. (Augenarzt Dr. Cause) ergab: Es besteht sehr hohe Hyperopie beiderseits; nach Ausgleich derselben gute Sehschärfe für Nähe und. Ferne. Gesichtsfeld zur Zeit absolut normal, Augenbewegungen intakt. Ophthalmoskopischer Befund ohne Veränderungen.

Die übrigen Hirnnerven, Motilität und Sensibilität normal, Haut- und Sehnenreflexe ungestört, die letzteren recht lebhaft, aber ohne-Klonus; Babinski und Oppenheim fëlen.

Körperliche Stigmata der Hysterie fehlen.

Während der kurzen Beobachtungszeit machte Pat. psychisch zwar einen ruhigen Eindruck, aber erweckte doch den Verdacht einer "stillen" Hysterie (als solche galt sie auch dem Hausarzt).

Migräneanfälle kamen - während der kurzen Beobachtung - leider nicht vor. Gegen ärztlichen Rat verliess Pat. vorzeitig dàs Spital.

Der Fall hat in mehrfacher Beziehung Interesse. Einerseits ist er ein weiteres Beispiel zu den seltenen Fällen, in denen die visuelle Aura des Flimmerskotoms allmählich durch stereotype Gesichtshalluzinationen verdrängt wird.

Die Angenmigräne der Kranken ging augenscheinlich mit einer ganz charakteristischen Hemianopsie einher. Die Angabe, dass die Skotomhälfte eine dunkle, rote Farbe habe, ist gerade bei der Hemianopsie der Migräne nicht ungewöhnlich. Ausserdem gingen diese Augenstörungen mit: vorübergehender Augenmuskellähmung einher; die horizontalen Doppelbilder sprechen für eine periodische Abducensparese.

Es war nun sehr interessant, dass die im Anfall eintretende Hemianopsie nicht nur an den realen Gegenständen der Umgebung (z. B. den Gesichtiern), sondern auch an den stereotypen rein halluzinierten Fratzen mit verstummelten Nasen and Ohren das typische hemianopische Skotom hervorrief. Ich weiss nicht, ob schon genügend auf analoge: Vorgänge- geachtet worden ist, glaube aber, dass eine derartige Übertragung einer körperlich gestörten Sehfunlition auf ein halluziniertes Objekt höchst selten ist. Dass entsprechende Vorgänge anch im Traume selten sind, wissen wir. Hier sind fast stets körperlich gestörte Funktionen kompensiert und werden beim Erleben des Traumbildes ausgeschaltet (vgl. die lebhaften visuellen Träume vieler Blinder).

Bei unserer Pat: "ist die Übertragung des (hemikranisch ausgelösten) hemianopischen Skotoms auch auf die halluzinierten Gesichter deshalb erklärlich, weil die Hemianopsie erheblich älteren Datums war als das Auftreten der stereotypen Hallazination; die erstere 'ging der 
letzteren ca. 10 Jahre voraus. Der Begriff und der Eindruck der hemianopischen Störung des Gesehenen hatte sich also derartig fest mit dem Auftreten des Migräneanfalls verkettet, dass durch diese feste Assoziation, durch die Gewöhnung an die Hemianopsie, diese Störung auch ganz mechanisch, wie selbstrerständlich auf die halluzinierten Gesichter übertragen wurde.

Andererseits "steckte" diese Halluzination auch die Bilder der realen Umgebung "an": auch die Gesichter der wirklichen Menschen (die Pat., wie bemerkt, scharf von den rein halluzinierten unterscheiden kann) erhielten im Beginn des Anfalls Anklänge von Verzerrung und Verstümmelung wie die rein halluzinierten.

Von Interesse war die Entstehung dieser visuellen Aura. Erst nach längerem eindringlichen Befragen gab Pat. an, dass sie kurz vor der Entstehung dieser Halluzination (also vor ca. 5 Monaten) eines Tages plötzlich auf der Strasse (oder in der Strassenbahn) dem Anblick einer Person mit augenscheinlich lupös zerfressener Nase und verstümmeltem $\mathrm{Ohr}$ ausgesetzt worden sei und den Anblick nicht habe loswerden können. Dieser (durch die starke Gefühlsbetonung des Mitleids besonders fest fixierte) Eindruck haftete seitdem, sie sah ihn auch im Traum - und eines Tages ging er als stereotype Halluzination in den Migräneanfall über.

Bemerkenswert ist, dass Pat., nachdem ibr dieser psychogene Zusammenhang zwischen Erlebnis und Halluzination klar geworden ist, bemerkt, sie glaube, dass auch die eigentümlich rötliche Färbung des hemianopischen Skotoms (wie sie sie besonders bei Gesichtern bemerkt) entschiedene Ähnlichkeit mit den grossen "Feuermalen“ (Naevus) mancher Menschen habe; sie erinnere sich, früher auch einmal einen derartig veranstalteten Menschen lange gesehen und sich vor ihm geekelt zu haben. Vielleicht sei dieser Eindruck nun auch schuld an der Lokalisierung und Färbung der Skotome auf den Gesichtern der (realen) Menschen.

Ich brauche nicht zu betonen, dass diese Annahme augenscheinlich irrig ist und eine Art nachträglicher „Volksetymologie" darstellt.

Der Fall ist auch dárum von klinischem Interesse, weil die stereotypen Halluzinationen bei unserer Kranken nicht mit Bewusstseinsveränderungen oder epileptischen Zuständen, überhaupt nicht mit gröberen psychischen Störungen einhergingen, im Gegensatz zu den (wenigen) analogen Fällen der Literatur.

Diese Fälle der Literatur gehören fast durchweg in das Gebiet der Hemicrania ophthalmica, d. i. der Migräneform, bei der regelmässig wiederkehrende Sehstörungen im Vordergrund der Symptome stehen. Diese Halluzinationen können der Augenmigräne auch schein- 
bar vorausgehen in Gestalt von visuellen Traumbildern. Féré hat diese „rêves précurseurs" besonders geschildert, u. a. das regelmässige Erscheinen einer weissen Gestalt im Traum; nach dem Erwachen begann dann stets die ophthalmische Migräne.

Ich glaube, dass diese stereotypen Traumbilder weniger als Prodrome anzusehen sind, sondern schon als echt visuelle Aura des Anfalls, genau wie in unserem Fall. Es ist ja allgemein bekannt, dass bei nicht wenigen Hemikranikern der Anfall immer im Schlaf, besonders im Tagessehlaf, beginnt. Die Patienten haben dann schon während des - bei ihnen manchmal sehr protrahierten - Stadiums des Halbschlafs vor dem Erwachen das immer bestimmter werdende Gefübl der Migräne.

Meist sind die in der Literatur bekannten Fälle von Halluzinationen im Migräneanfall nicht so stereotyp wie in unserem Fall, sondern wechselnder. Z. B. sah eine 55jähr. Pat. Kraft-Ebbings im Anfall ihrer hemianopischen Migräne Fratzen, Statuen, Pagoden in ständiger Bewegung; danach Bewusstseinsverlust. In einem Fall von Bordoni sah die Pat. Tiere, Männer mit Messern, abgeschnittene Köpfe, hörte Stimmen a. dgl.; daneben typische hysterische Anfälle.

Durchaus stereotype Gesichtserscheinungen zeigen, soweit ich aus der Literatur ersehe, nur wenige Fälle, z. B. der von Mingazzini: In einem Fall von linksseitiger Augenmigräne kommt es stets zur Vision eines schwarzen Mannes, der drohend auf den Pat. losgeht, ihn packt; dann kurzer Bewusstseinsverlust. ב̈hnlich ist ein Fall von J. K. Mitchell: Einer Pat, erscheint unter zunehmender Amblyopie im Beginn des Migräneanfalls regelmässig die Gestalt eines Zwerges mit einer Kenle, der die Pat. schlägt; auch hier endet der Anfall mit Bewusstseinsverlust und Konvulsionen anscheinend hysterischen Charakters.

Dass nicht nur die essentielle Migräne, sondern auch die sekundäre Form bzw. die symptomatische Migräne zu stereotypen Halluzinationen führen kann, lehrte mich folgende eigene Beobachtung der jüngsten Zeit:

Fall 5. Bei einem 13jähr. Sclüler bestehen seit ca. 2 Monaten Anfälle von linksseitigem „Nervenkopfweh“.

Anfangs begannen sie mit einem typischen Flimmerskotom unter leichter Verdunkelung des rechsseitigen Gesichtsfeldes. Dieses Flimmerskotom macht nun, seitdem die Anfälle seit 3 Wochen viel häufiger, jeden zweiten bis dritten Tag kommen, einer ganz stereotypen visuellen Erscheinung Platz: Eiin weisses Männchen, das anfangs verschwommen und klein, später deutlich und gross wurde, bewege sich unter rhythmischen Bewcgungen "nur vor dem rechten Auge“. Dabei zeigt der Junge laut Angabe der Mutter - keine Spur von hysterischen oder epileptischen 
Erscheinungen im Anfall. Nur einmal erlitt er eine tiefe Ohnmacht im Anschluss an den Anfall.

Der organische Befund war anfanys völlig normal. Jetzt wird eine Staungspapillitis beiderseits $(\mathbf{r} .>1$.) immer deutlicher, der Junge verfällt sichtlich, leidet oft an heftigen Kopfschmerzen, hat bisweilen Temperaturen. Die Diagnose eines Tumors noch unbekannten Sitzes ist demnach leider das wahrscheinlichste.

Auch nach spezialistischer Untersuchung (Dr. Cause-Mainz) spricht der Augenbefund für einen Tumor: „Beiderseits deutliche Staungspapille bei noch normaler Sehschärfe (geringe Hyperopie), normale Aussengrenzen des Gesichtsfeldes für Weiss und geringe konzentrische Einschränkung für Farben". Die Untersuchung wurde an einem Tage gemacht, an dem Kopfdruck und -schmerz relativ gering waren.

Übrigens hat man auch aus dem Auftreten optischer Halluzinationen auf den Sitz eines Tumors schliessen wollen. Es werden bei Tumoren im Bereich der Sehsphäre des Hinterhauptlappens bisweilen flimmerskotomartige Gesichtsbilder beschrieben. In unserem Falle glaube ich das Auftreten der stereotypen Halluzination aber darum topisch nicht verwenden zu dürfen, weil sie nicht mit permanenter Hemianopsie, sondern nur mit einer geringen konzentrischen Einengung des Gesichtsfeldes für Farben verbunden ist. Die rechtsseitige Hemianopsie während des Migräneanfalls ist natürlich keinesfalls lokaldiagnostisch mit einiger Sicherheit zu verwenden.

Ich möchte übrigens hinzufügen, dass dies Flimmerskotomsymptom bei Erkrankungen der Sehsphäre doch selten und auch darum topisch wenig verwendbar erscheint. Ich habe es wenigstens weder in einem länger beobachteten Fall von rechtsseitigem Occipitaltumor mit Danerhemianopsie (Diagnose durch Operation bestätigt) gesehen, noch in drei Fällen von Hintexhauptschüssen mit anfänglich schweren Sehstörungen. Einer der letzten Pat., ein Major, hatte zwar Anfälle von heftigem Kopfschmerz mit Pulsverlangsamung und Erbrechen, aber ohne jede visuelle oder gar halluzinatorische Aura.

Dasselbe gilt von einem Soldaten mit schwerer stumpfer Gewalteinwirkung auf das Occiput und anfänglich totaler Rindenblindheit. Auch dieser Pat., den ich seit vielen Wochen klinisch beobachte, hatte niemals visuelle Skotome.

Einer kurzen Besprechnng bedürfen noch gewisse Erfahrungen, die ich, wie bereits erwähnt, bei der Migräne der Kinder gemacht babe. Es ist nicht zu viel behauptet, wenn ich sage, dass das erste Auftreten, insbesondere der erste Anfall der Migräne bei einem Kinde überhaupt sehr häufig diagnostisch verkannt wird und dass sich aus diesen Fehldiagnosen üble therapeutische Missgriffe entwickeln können. Dass der „erste Anfall“ tatsächlich diagnostische Schwierigkeiten machen kann, ist zuzugeben, zumal er sich, was in der Literatur 
nicht genügend betont wird, gar nicht selten auch an Infektionskrankheiten anschliesst, infolgedessen also als Komplikation oder Nachkrankheit derselben imponieren kann.

Zwei besonders charakteristische Fülle meiner Beobachtung mögen dies belegen.

Fall 6. Kindermigräne unter dem Bilde der Meningitis.

$\mathrm{Zu}$ dem Kind M. H., $10 \mathrm{~J}$, Tochter nervöser Eltern, wurde ich konsultiert mit der Bitte des Hausarztes, das Lumbalpunktionsbesteck mitzubringen, da wahrscheinlich eine Meningitis vorliege.

Das bisher gesunde Kind war vor ca. 5-6 Tagen an typischer Influenza (Familieninfektion) erkrankt mit mässigen Temperaturen, Schnupfen, Husten usw. Seit 2 Tagen wieder wohl und fieberfrei, erkrankte es nach kurzen Prodromen mit enorm heftigen Kopfschmerzen, besonders im Hinterkopf, leichter Benommenheit, mehrfachem Erbrechen und allgemeiner Unruhe.

Fieber bestand nicht. Ein sofort zugezogener Ohrenarzt schloss einen othogenen Prozess aus.

Da der Zustand sich innerhalb 24 Stunden nicht besserte und sehr bedrohlich erschien (der Arzt teilte den Eltern die Befürchtung der Meningitis mit!), wurde ich zugezogen.

Bei der Untersuchung ging es dem Kind schon etwas besser, es gab nun einen fast rein halbseitigen Kopfschmerz, besonders in der Nackenand Schläfengegend, an; die gleichseitigen Halsmuskeln waren anf Druck sehr schmerzhaft. Eine wirkliche Nackensteifigkeit bestand nicht, ebensowenig das Kernigsche Phänomen. Tonus der Beine normal, desgleichen die Sehnenreflexe; kein Babinski, keine Hyperästhesie der Waden and Fusssohlen, kein Kahnbauch, keine Bradykardie.

Pupillen und Augeñmuskeln intakt. Augenhintergrund o. Ver.

Psychisch war das Kind auch wieder ganz normal.

Die Familienanamnese ergab, dass sowohl die Mutter und Grossmutter, als andere, besonders weibliche Angehörige an typischer Migräne litten, die bei der Mutter auch schon in der Schulzeit begonnen hatte.

Dementsprechend stellte ich die Diagnose auf den „ersten Migräneanfall" des Kindes, verordnete Phenacetin-Coffein und Ruhe, und am Morgen des nächsten Tages war das Kind wieder gesund und völlig beschwerdefrei.

Wie ich höre, hat das Kind auch späterhin an typischem „Nervenkopfweh" gelitten.

Ein weiterer erst kürzlich beobachteter Fall verlief ganz ähnlich.

Fall 7. Migräneanfall in der Scharlachrekonvaleszenz, Pseudourämie.

Die 11jähr. Tochter des Hauptmann R. sah ich zuerst am 9. VI. 1915 wegen schwerer Scarlatina; der Fall war bemerkenswert dadurch, dass das Kind bereits im vorigen Jahre eine absolut sichere Scarlatina durchgemacht hatte. Der Fall schien ganz normal zu verlaufen, Entfieberung zur normalen Zeit, starke Schuppung.

Da trat am 30. Juni nach Angabe der Eltern eine schwere Ver- 
schlimmerung des Zustandes ein, der die Eltern veranlasste, das Kind aus der hausärztlichen Behandlang in meine klinische Behandlung zu überführen, da sie annahmen, dass unmittelbare Lebensgefahr bestände.

Das Kind hatte am 30. früh über allgemeine Mattigkeit, Appetitlosigkeit und zuerst links-, dann auch rechtsseitigen Stirnkopfschmerz von zanehmender Heftigkeit geklagt; darauf sei zuerst das linke, später das rechte Auge stark angeschwollen. Es traten Übelkeit und Erbrechen ein. Das Kind war ausserordentlich blass und elend, teilnabmslos bis zur Somnolenz, machte einen anscheinend schwerkranken Eindruck. Der Hausarzt sprach (vor der Untersuchung des Urins) angesichts der vorausgegangenen Scarlatina und der bestehenden Lidödeme, der Benommenheit und des Erbrechens von schwerer akuter Nephritis und beginnender Urämie. Darauf sofortige Überführung ins Krankenhans.

Bei der Untersuchung am 1. VII. ging es dem Kind schon besser. Das doppelseitige Lidödem war verschwunden, das Kind war blass, nicht gedunsen. Starke scarlatinöse Schuppung. Es besteht noch leichter Kopfschmerz, Druckschmerz der Supraorbitalpunkte, Klopfschmerz des Schädels; keine Nackenstarre, kein Kernig.

Der Urin war frei von Eiweiss und Blat; mikroskopisch keine Zellen, keine Zylinder.

Herz und Atmungsorgane gesund.

Am Nervensystem ebenfalls keine organischen Veränderungen.

Das Kind war am nächsten Tag wieder ganz beschwerdefrei, ohne Kopfweh, hatte vorzüglichen Appetit.

Die Familienanamnese ergab hier eine doppelte spezifische Belastung: Der Vater litt an häufigen, ziemlich schweren Anfällen von Hemikranie mit Flimmerskotom, Erbrechen usw.; die Mutter litt an seltenen Anfällen von halbseitigem Nervenkopfweh mit Übelkeit, meist ohne Erbrechen. In der Familie des Vaters noch weitere schwere Hemikraniefälle.

In beiden Fällen ist die Diagnose des exsten Migräneanfalls unzweifelhaft. Der heftige überwiegend halbseitige Kopfschmerz (ohne Fieber), die Übelkeit, das Erbrechen, die scharfe zeitliche Begrenzung des Anfalls, das Lidödem im zweiten Fall, die später repetierenden Migräneanfälle im ersten Fall und die ausgesprochene Migräneheredität sichern in beiden Fällen die Diagnose.

Bemerkenswert war bei beiden Kindern das erste Auftreten nach einer akuten Infektion, nach Influenza bzw. Scharlach; die Ursache, weswegen beide Fülle anfangs missgedeutet und als Meningitis und Urämie aufgefasst worden waren.

Es ist, wie schon bemerkt, auffallend, wie wenig die Rolle der akuten Infektionskrankheiten als auslösende Ursache von Neurosen, vor allem bei Kindern und Jugendlichen im allgemeinen beachtet wird. Das gilt sowohl von mannigfachen Äusserungen der Hysterie als auch von der Migräne.

Auch Flatau bemerkt mit Recht, dass das bisherige kasuistische Material nach dieser Richtung noch sehr dürftig erscheint und dass 
es lohnend sei, entsprechende Studien durchzuführen. Finige wenige Beispiele von Erstanfällen nach Typhus und Scharlach (Möbius), Malaria (Gowers, Trousseau) und Influenza (Flatau) erwähnt Flatan.

Ich habe noch weitere Fälle gesehen, die ibre ersten Anfälle nach Diphtherie, Scharlach und endlich Typhus erlitten hatten.

Charakteristisch ist in allen diesen Fällen das Auftreten in der fieberfreien Rekonvaleszenz; im Fieberstadium einer akuten Infektionskrankheit babe ich, soweit ich mich entsinne, auch bei schweren Hemikranikern niemals einen Anfall beobachtet; ebenso wie während desselben ja auch Epileptiker von ihren Anfällen versehont zu bleiben pflegen.

Nicht nur das Auftreten direkt nach einer Infektionskrankheit, sondern auch die Schwere und relativ lange Dauer des Migräneanfalls hatte in beiden Fällen den vorbehandelnden Ärzten die Diagnose verschleiert. Fs ist eben eine viel zu wenig bekannte Tatsache, dass der erste Anfall der Migräne im Jugendalter von ungewöhnlich schwerer und langdauernder Art sein kann. Auch in den bekannten Migränemonographien (Flatau, Möbius u. a.), die überhaupt die Kindermigräne etwas stiefmütterlich behandeln, finde ich diese Tatsache nicht erwähnt, ebensowenig in der Bearbeitung der Migräne von Thiemich (im Pfaundler-Schlossmannschen Handbuch ${ }^{1}$ )). Gerade aus diesem Kapitel, in dem die Kindermigräne in einer Seite abgetan wird, während der allerdings aktuelleren und die zünftigen Pädiater mehr beschäftigenen Spasmophilie-Tetanie ca. 26 Seiten gewidmet sind, sieht man zur Genüge, dass die Hemikranie des Kindes von den Pädiatern etwas kurz, jedenfalls nicht ihrer Bedeutung entsprechend behandelt wird. Es ist sehr kennzeichnend, wenn Thiemich nach eigenen Erfahrungen an der Breslaner Kinderklinik von der Seltenheit der Hemikranie im Kindesalter spricht. Ich glaube aber nicht, dass diese anderen gegenteiligen Anschauungen gegenüber betonte Seltenheit der Migräne durch lokale Unterschiede bedingt ist, wie Thiemich meint, sondern bin der Ansicht, dass gerade für die Migränebeobachtnng auch im kindlichen Alter nicht das klinische Krankenmaterial, sondern das der Privatpraxis die grösste Ausbeute gibt; es ist mit der Migräne der Erwachsenen iibrigens ganz ebenso.

Man frage nur die schärfer beobachtenden Mütter weniger Kinder (d. i. die der Praxis melior), wenn sie über Hemikranie klagen, nach etwaigen nervösen Symptomen ihrer Kinder, insbesondere derjenigen des Spiel- und Lernalters, aus. Aus ihren Antworten wird man rich-

1) Bd. 4 des Handbuchs S. 349 . 
tige Schlüsse auf die Häufigkeit und Beginn der Kindermigräne ziehen können, nicht aber aus dem stummen Krankenmatérial der Klinik.

Ich lasse nun zum Sehluss zwei bemerkenswerte Beobachtungen über symptomatische und echte Migräne in ihrem Verhältnis zur multiplen Sklerose folgen.

Fall 8. Symptomatische Augenmigräne als Frühsymptom der multiplen Sklerose.

F. F., 19jähr. Ladnerin aus W., der Abteilung auf kurze Zeit zum Zweck eines Obergutachtens überwiesen. Der vorbegutachtende Arzt hatte wegen Hysterie and Migräne ein Heilverfahren beantragt.

Die Eltern seien gesund, eine Schwester rheumatisch, keine Nervenoder Geisteskrankheiten, Epilepsie usw. in der Familie. Als Kind stets gesund, keine Rachitis, gute Schülerin. Mit 131/2 Jahren menstruiert, bisher stets regelmâssig. Seit dem 16. Jahr Verkäuferin in grossem Warenhaus. An Sehstörungen, Doppeltsehen oder anderen Augenerscheinungen will sie nie gelitten haben.

Im September 1913 stürzte sie ,aus Unvorsichtigkeit", nicht etwa weil sie schon unsicher anf den Beinen war, die Treppe herunter, ohne sich zu verletzen; sie konnte gleich weiterarbeiten. Seit dieser Zeit will sie nun häufig an Anfällen von Kopfweh leiden, das alle 3-8 Tage auftrat, über dem linken, seltener über dem rechten Auge oder der entsprechenden Scheitelseite sass, mit etwas Übelkeit, aber obne Erbrechen und ohne Augenstörungen einherging. Am 21. März wurde ihr plötzlich schlecht. Sie bekam heftigen Schwindel, Kopfschmerzen, Übelkeit mit Erbrechen und starke Schmerzen im rechten Bein mit Taubheit derselben, geringere Schmerzen und Taubheit auch im rechten Arm. Sie legte sich ins Bett. Der heftige halbseitige (rechtsseitige) Kopfschmerz mit starkem Erbrechen - oft 14-16 mal am Tag - dauerte 12-14 Tage. Dann nahmen Kopfweh und Erbrechen ab. Als sie nach ca. 3 Wochen wieder aufstand, war das rechte Bein schwach und ungeschickt; sie konnte nur schlecht gehen. Der rechte Arm hatte sich rasch gebessert.

Bis Oktober 1914 war sie nun fast wieder hergestellt. Das rechte Bein funktionierte wieder ganz gut. Nur ziemlich seltene Anfälle von halbseitigem Kopfschmerz mit etwas Übelkeit und seltenem Erbrechen, anscheinend typische Migräne, blieben zurück.

So blieb der Zustand bis zum Oktober 1914. Da wiederholte sich das Leiden vom vorigen Jahr, aber in etwas anderer, mehr anfallweise intermittierender Form: Während das rechte Bein wieder schwächer wurde, traten nun alle 3-4 Tage Anfälle von heftigem, meist rechtsseitigem Kopfschmerz a uf, die regelmässig mit starker Übelkeit und Erbrechen einhergingen. Eingeleitet wurden diese "Migräneanfälle" durch Doppeltsehen bei Blickrichtung nach rechts; „die Gegenstände standen nebeneinander, nicht schief, sondern in einer Höhe", und Verdunkelung der rechten Seite der angeschauten Gegenstände (z. B. besonders deutlich an Gesichtern, deren eine Seite grau und unscharf begrenzt erschien).

Diese Anfälle wiederholten sich nun alle $3-4$ Tage bis zum Dezember 1914, gingen stets mit einleitender Diplopie und Hemianopsie einher und endigten stets mit Erbrechen. Das Sehvermögen sei im ganzen schlechter 
geworden. Auch das rechte Bein wurde wieder etwas tauber und ungelenker, infolgedessen der Gang etwas unsicherer, mehr subjektiv als objektiv.

Seitdem habe sich unter energischer ärztlicher Behandlung alles gebessert. Nur die in unregelmässigen Intervallen, seltener als früher auftretenden Anfälle von halbseitigem, seltener Scheitelkopfschmerz seien geblieben. Dieselben seien stets mit Doppeltsehen bei Blick nach rechts verbunden, die Verdunkelung des rechten Gesichtsfeldes sei nicht mehr so ausgesprochen wie früher. Die Anfälle gingen noch immer mit Übelkeit einher, aber nur noch selten mit Erbrechen.

Status (April 1915). Kräftig gebautes, etwas anämisches Mädchen, das bei oberflächlicher Beobachtung alles andere eher als eine organische Spinalerkrankung vermuten lässt.

Innere Organe ohne pathologischen Befund.

Pupillen gleich, von normaler Konvergenz- and Lichreaktion. Zur Zeit keine Augenmuskelparesen (es wird auch in der stets anfallfreien Zeit der Beobachtung nie über Doppeltsehen geklagt), jedoch starker Nystagmus horizontalis bei Blickrichtung nach rechts, d. i. die Richtung, bei der sonst über horizontale Doppelbilder geklagt wird. Geringerer, aber auch konstanter Nystagmus nach links. Augenhindergrund: Keine sichere Veränderung der Papille. Perimetrium: Zur Zeit für Weiss und für Farben normal (Augenarzt Dr. Deters). Untersuchung ebenfalls in anfallfreier Zeit.

Alle Hirnnerven, besonders Facialis, Hypoglossus, ohne Veränderung.

Motilität: Beim Gehen mit blossen Füssen fällt eine geringe, aber deutliche spastische Ataxie des rechten Beins auf, besonders nach längerem Gehen. Bei Balancierübungen ohne Augenkontrolle auch anffallende allgemeine Unsicherheit des Ganges. Rombergsches Phänomen positiv. Geringes Intentionszittern nur der rechten Hand (nicht konstant).

Sensibilität: Hypästhesie besonders für Berührungs- und Schmerzreize, kaum für Warm und Kalt am rechten Fuss and Unterschenkel nach oben zu ganz unscharf begrenzt; an den Fingern der rechten Hand nur subjektive leichte Hypästhesie. Störungen der Stereognosie and des Bewegungsgefühls fehlen.

Reflexe: Unterkieferreflex erhöht.

Alle Sehnen- und Periostreflexe der oberen Extremitäten erhöht, r. $>1$., Jakobsohnsches Phänomen rechts positiv.

Patellar- und Achillessehnenreflexe beiderseits stark gesteigert, rechts Patellar- und Fussklonus.

Rechts konstant typischer Babinski, links meist dasselbe, rechts und links K. Mendel-Bechterewsches Zeichen positiv. Oppenheim, Remak und Strümpells Tibialisphänomen fehlen.

Bauchdeckenreflexe: Nur der rechte untere ist häufig vorhanden, aber auch rasch erschöpfbar, die übrigen fehlen.

Blase und Mastdarm o. Ver.; Periode o. Ver. - Vasomotorische und trophische Störungen fehlen.

Psyche: Lebhafte, intelligente, meist recht vergnügte Pat.; völlig unhysterisches Verhalten. Auffallend war nur - bei dem Fehlen eigentlicher Zwangsaffekte - eine gewisse "reizbare Affektschwäche". Sie geriet relativ leicht in Erregung und drohte zu weinen, konnte aber ebenso leicht „unter Tränen lächeln". Im ganzen trug sie ihr Leiden mit einer an- 
gesichts der objektiven Krankheitszeichen das normale Maß überschreitenden Euphorie.

Der Verlauf des Falles hat durch den auffallenden Erfolg der siebenwöchentlichen Fibrolysinbehandlung, die wir nach M. Nonnes Vorgang anwendeten, Interesse: Pat. besserte sich in jeder Beziehung ausserordentlich. Der Gang wurde ganz normal, sogar die Bauchdeckenreflexe kehrten wieder, zuerst verschwand der linksseitige Babinski, später sogar der (bis dahin konstante) rechtsseitige Babinski. Auch die migräneähnlichen Kopfschmerzen verschwanden völlig. Alles in allem: eine der schönsten Remissionen und sicher die rascheste, die ich bei der Behandlung einer multiplen Sklerose gesehen habe.

Epikrise: Uns interessiert im Krankheitsverlauf dieser im Untersuchungsbefund typischen initialen multiplen Sklerose vor allem die Phase vom Oktober 1914 bis zum Eintritt in die Behandlung April 1915.

Während bald nach dem Trauma uncharakteristische Kopfschmerzen und im März bis April 1914 ein akuter initialer "Schub" mit dem üblichen schweren Status cerebralis (Kopfweh, permanentes Erbrechen usw.) auftraten, bestanden seit Oktober 1914 bei der Pat. Kopfbeschwerden, die absolut einer echten Augenmigräne glichen und - zumal sie ganz im Vordergrund der Beschwerden standen auch vom Arzt so gedeutet wurden. Das Auftreten einer echten Migräne neben der angenommenen Hysterie und der tatsächlich bestehenden Chlorose hatte ja auch bei einem erst 18jährigen Mädchen nichts Auffallendes.

Die geschilderten Anfälle verliefen regelmässig mit rechtsseitigem Kopfschmerz, starker Übelkeit und Erbrechen und wurden eingeleitet durch horizontale Diplopie and ein hemianopisches Skotom, sie repetierten anfangs alle 3-4 Tage, später in den letzten 4 Monaten in unbestimmten Abständen wesentlich seltener.

Mit der Beseitigung der übrigen subjektiven and objektiven Symptome der multiplen Sklerose verschwanden anch die Anfälle.

Die Anfälle waren also scheinbar so typisch für echte Migräne, dass vielleicht der Einwand gemacht werden könnte: Handelt es sich nicht etwa um eine blosse zufällige Kombination beider Krankheitszustände; eine Möglichkeit, die bei der Häufigkeit beider Leiden ja nicht ausgeschlossen wäre.

Dagegen spricht aber die Entstehung dieser scheinbaren Migräne: sie erwuchs nämlich aus einem typischen initialen Schub der multiplen. Sklerose, der mit demselben ausschliesslich rechtsseitigen Kopfweh begann, ebenfalls zu Erbrechen führte, aber dann unter allgemeinen schweren Gehirnsymptomen 12-14 Tage dauerte und eine deutliche sensible und motorische Parese des rechten Beins (eine geringe auch des rechten Arms) von längerer Dauer hinterliess. 
Auch die Tatsache, dass der zur Zeit bestehende Nystagmus fast nur bei Blickrichtung nach der Seite auftritt, bei der im Anfall zweifellos eine Abducenslähmung eintritt, ist ein wichtiges Moment für die grob organische Natur dieses Symptoms; dieser Nystagmus ist eben der typische "Schwächenystagmus", wie er als Rest einer organischen Augenmuskellähmung gerade bei multipler Sklerose sehr häufig vorkommt; bei Angenmigräne findet man ihn aber sicher nur selten.

Auch das Verschwinden der scheinbaren Angenmigräne zugleich mit der heilungsähnlichen Remission der multiplen Sklerose spricht mit Bestimmtheit dafür, dass die erstere eben nur ein Symptom der letzteren war.

Die Pseudomigräne in dieser ansgeprägten Form ist als Frühsymptom ganz ausserordentlich selten. Die erfahrensten Kenner der Migräne (Möbius, Oppenheim, Flatau u. a.) erwähnen unter den zahlreichen Arten der symptomatischen Migräne die multiple Sklerose als Urheberin derselben überhaupt nicht.

Es ist die Frage, ob Fälle wie der meinige aber nicht doch etwas häufiger sind, als nach Fehlen einschlägiger Mitteilungen anzunehmen wäre. Wenigstens spricht die Bemerkung Eduard Müllers dafür, dass man zuweilen intensiven, einseitigen und paroxysmal auftretenden Kopfschmerz bei multipler Sklerose finden könne, der „bei flüchtiger Untersuchung den Verdacht auf Migräne erwecken könne". Allerdings bringt auch E. Müller keinen Fall eigener oder fremder Beobachtung, der so genau das Symptomenbild der Augenmigräne imitiert hätte, wie dies der unsrige Fall getan hat.

Bei der ungemein häufigen Verkennung der Frühstadien der multiplen Sklerose und ihrer fast stets erfolgenden Verwechslung mit allerlei fnnktionellen Neurosen ist es also wichtig, durch den obigen Fall zu lernen, dass das Initialstadium der multiplen Sklerose mit einer scheinbar reinen Augenmigräne verlaufen kann. Man wird also auch derartige Fälle etwas genauer auf die Symptome der multiplen Sklerose untersuchen müssen. Vielleicht wird man dann finden, dass das von mir geschilderte Symptomenbild bei multipler Sklerose häufiger ist, als bisher vermutet.

Im Hinblick auf das Eintreten einer symptomatischen Migräne als Frühzeichen der multiplen Sklerose ist nun ein unlängst beobachteter Fall recht bemerkenswert, der das Umgekehrte zeigt:

Die Heilung bzw. Beseitigung der Migräneanfälle durch das Auftreten einer multiplen Sklerose.

Fall 9. Frau B., 40 Jahre, leidet an einer recht typischen und schweren Form der multiplen Sklerose. 
Seit 2 Jahren sind allmählich Spasmen in beiden Beinen aufgetreten. Zur Zeit bestehen: spastische Paraparese der Beine mit geringer Ataxie, dabei Hyperreflexie der Beine, Babinski, Remak und Oppenheim positiv. Bauchdeckenreflexe fehlen.

Leichter Intentionstremor rechts. Augenbefund bis auf beiderseitigen Exophthalmus normal, speziell keine Veränderungen der Papillen. Nystagmus gering. Auffallende Neigung zur Euphorie und zum Lachen ohne eigentliche Zwangsaffekte. Kein Skandieren. Blasenlähmung. Nur sehr geringe sensible Störungen an den Füssen und den Fingern.

Die Periode blieb normal erhalten, keine Climax praecox.

Das uns hier besonders jnteressierende Moment ist die Migränean amnese der Pat.: Mutter litt an schwerer Migräne, starb geisteskrank; keine Geschwister. Pat. hatte seit früher Jugend, sicher seit der Schulzeit typische Anfälle von Nervenkopfschmerz über und in dem rechten Auge, das sich dann von selbst schliesst (Lidkrampf?). Auf dem rechten Auge im Beginn des Anfalls Funken- und Punktesehen. Während des Anfalls Anorexie, Übelkeit und Brechreiz, aber kein Erbrechen; keine Durchfälle. Dauer des einzelnen Anfalls sehr schwankend zwischem einem und drei Tagen. Die Anfälle traten mit grosser Regelmässigkeit während der Periode, bisweilen aber auch dazwischen auf.

Seitdem die spastische Parese der Beine, die Blasenschwäche und die allgemeine Zittrigkeit aufgetreten sei, also seit etwa 2 Jahren, ist die bis dahin absolut regelmässige Migräne völlig weggeblieben; auch während der Menses keine Andeutung von Kopfweh; auch kein "Ersatz" der Migräne durch Bauchkoliken, paroxysmale Diarrhöen, Herzanfälle u. dgl.

Der Fall ist deshalb interessant, weil er zeigt, dass auch die multiple Sklerose einmal einen ähnlichen tilgenden Einfluss auf die Migräne haben kann wie akute Infektionen, Stoffwechselkrankheiten oder auch physiologische Zustände, wie Schwangerschaft, Stillgeschäft und Wechsel. Es ist ja bekannt, dass die multiple Sklerose ihrerseits auf die Menstruation häufig eine starke Einwirkung hat und sogar nicht selten zur vorzeitigen Climax führt, die wiederum so häufig die Migräne zum Erlöschen bringt. In unserem Fall hat das Rückenmarksleiden die Genitalfunktion intakt gelassen, dafür aber die Migräne beseitigt.

Ich hoffe durch die geschilderten Beobachtungen aufs neue einiges Interesse für die merkwürdige Mannigfaltigkeit der eminent „friedlichen" Hemikranie - unter unseren erkrankten Feldzugsteilnehmern, auch den nervösen, spielt sie in der Tat eine auffallend geringe Rolle - erweckt und gezeigt zu haben; dass selbst einem so alltäglichen und literarisch ausgeschlachteten Leiden noch symptomatologisches Interesse abzugewinnen ist, wenn man seine Betrachtung um einige allgemein pathologische Punkte erweitert. 